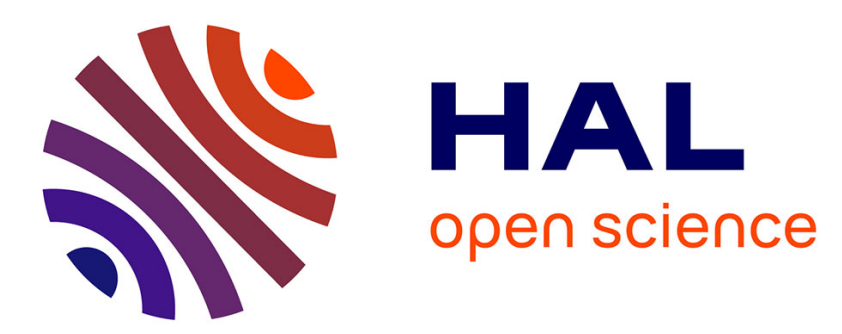

\title{
Transitions de phases et modes d'épitaxie complexes dans les couches minces d'oxydes de terres rares irradiées par un faisceau d'électrons
}

M. Gasgnier, G. Schiffmacher, P. Caro

\section{- To cite this version:}

M. Gasgnier, G. Schiffmacher, P. Caro. Transitions de phases et modes d'épitaxie complexes dans les couches minces d'oxydes de terres rares irradiées par un faisceau d'électrons. Revue de Physique Appliquée, 1988, 23 (7), pp.1341-1344. 10.1051/rphysap:019880023070134100 . jpa-00245951

\section{HAL Id: jpa-00245951 https://hal.science/jpa-00245951}

Submitted on 1 Jan 1988

HAL is a multi-disciplinary open access archive for the deposit and dissemination of scientific research documents, whether they are published or not. The documents may come from teaching and research institutions in France or abroad, or from public or private research centers.
L'archive ouverte pluridisciplinaire HAL, est destinée au dépôt et à la diffusion de documents scientifiques de niveau recherche, publiés ou non, émanant des établissements d'enseignement et de recherche français ou étrangers, des laboratoires publics ou privés. 


\title{
Transitions de phases et modes d'épitaxie complexes dans les couches minces d'oxydes de terres rares irradiées par un faisceau d'électrons
}

\author{
M. Gasgnier, G. Schiffmacher et P. Caro \\ CNRS Bellevue, ER 060210, 1 place A. Briand 92125 Meudon Cedex, France
}

(Reçu le 26 janvier 1988, révisé le 11 avril 1988, accepté le 12 avril 1988)

\begin{abstract}
Résumé. - Les couches minces d'oxyde de terres rares sont des matériaux très particuliers. La série des oxydes intermédiaires de cérium, praséodyme et terbium en est une parfaite illustration. Les films traités à la vapeur d'eau, puis irradiés par un faisceau d'électrons sont le siège de transition de phases et de modes d'épitaxie complexes inattendus. Un processus d'ondes de chocs, induit par l'impact du faisceau au travers du matériau pourrait expliquer ces phénomènes.
\end{abstract}

\begin{abstract}
Rare earth oxide thin films are peculiar materials, especially in the case of the intermediate oxide series for cerium, praseodymium and terbium. Treatment by water-vapor and irradiation by means of an electron beam induce intricate transition phases and unexpected epitaxial modes. These phenomena are tentatively linked to shock-wave processus throughout the material.
\end{abstract}

\section{Introduction.}

Dans un microscope électronique, le traitement thermique des couches minces à l'aide du faisceau électronique permet d'observer des phénomènes de cristallisation et/ou de recristallisation et de transitions de phases souvent inattendus. Récemment, nous avons tenté d'expliquer certains processus de transitions structurales en considérant que l'impact d'un faisceau électronique pouvait donner naissance à des ondes de choc de forte intensité dans le matériau. La présence d'impuretés (vapeur d'eau, oxygène, ...) pourrait être un facteur amplificateur non négligeable dans le processus de production de ces ondes élastiques $[1,2]$.

\section{Procédé expérimental.}

Les couches minces de terres rares métalliques (40$100 \mathrm{~nm}$ ) sont déposées, sur des supports en floatglass ou en $\mathrm{NaCl}$, par évaporation sous vide (creuset en tungstène, pression d'environ $7 \times 10^{-5} \mathrm{~Pa}$ ). Après décollement les films sont déposés sur des grilles de cuivre. Certaines sont soumises à un flux de vapeur d'eau pendant plusieurs heures. Les études sont faites par microscopie électronique (Jéol 100C). Les recuits (entre 300 et $2200 \mathrm{~K}$ ) sont effectués à l'aide du faisceau d'électrons (3).
3. Transitions de phases des oxides de cérium, praséodyme et terbium.

Dans la série des terres rares (R) trois d'entre elles (cérium, praséodyme et terbium) forment des oxydes de valence intermédiaire définis par les formules: $\mathrm{RO}_{x}(1.5 \leqslant x \leqslant 2)$ ou $\mathrm{R}_{n} \mathrm{O}_{2 n-2}(4 \leqslant n \leqslant \infty)$. Certains de ces composés sont bien connus $(n=4,7,9,10$, $11,12$ et $\infty)(n=4$ définit les sesquioxydes $\mathrm{R}_{2} \mathrm{O}_{3}$ et $n=\infty$ les dioxydes $\mathrm{RO}_{2}$ ). Les couches minces oxydées de $\mathrm{Ce}, \mathrm{Pr}$ et $\mathrm{Tb}$ ont révélé que les phases de type $\mathrm{RO}_{x}$ étaient beaucoup plus nombreuses que celles définies ci-dessus [4, 5]. Eyring et al. [6-8] ont observé des phénomènes semblables pour des microcristaux broyés. Dans le cas du praséodyme, après formation, soit des sesquioxydes (A$\mathrm{Pr}_{2} \mathrm{O}_{3}$ hexagonal ou $\mathrm{C}-\mathrm{Pr}_{2} \mathrm{O}_{3}$ c.c.), soit du dioxyde $\left(\mathrm{PrO}_{2}\right.$ c.f.c. $)$, les cristaux minces sont recuits à basse température. A l'impact du faisceau d'électrons les surfaces apparentes changent rapidement d'aspect dans un très petit domaine de température et les contours d'extinction se déplacent aléatoirement (Figs. 1a à 1c). En même temps, à chaque changement correspond une nouvelle structure du cristal (Figs. 1d à 1f). Des cycles de chauffage et de refroidissement lents engendrent une multitude de phases métastables connues ou inconnues. Les cycles 

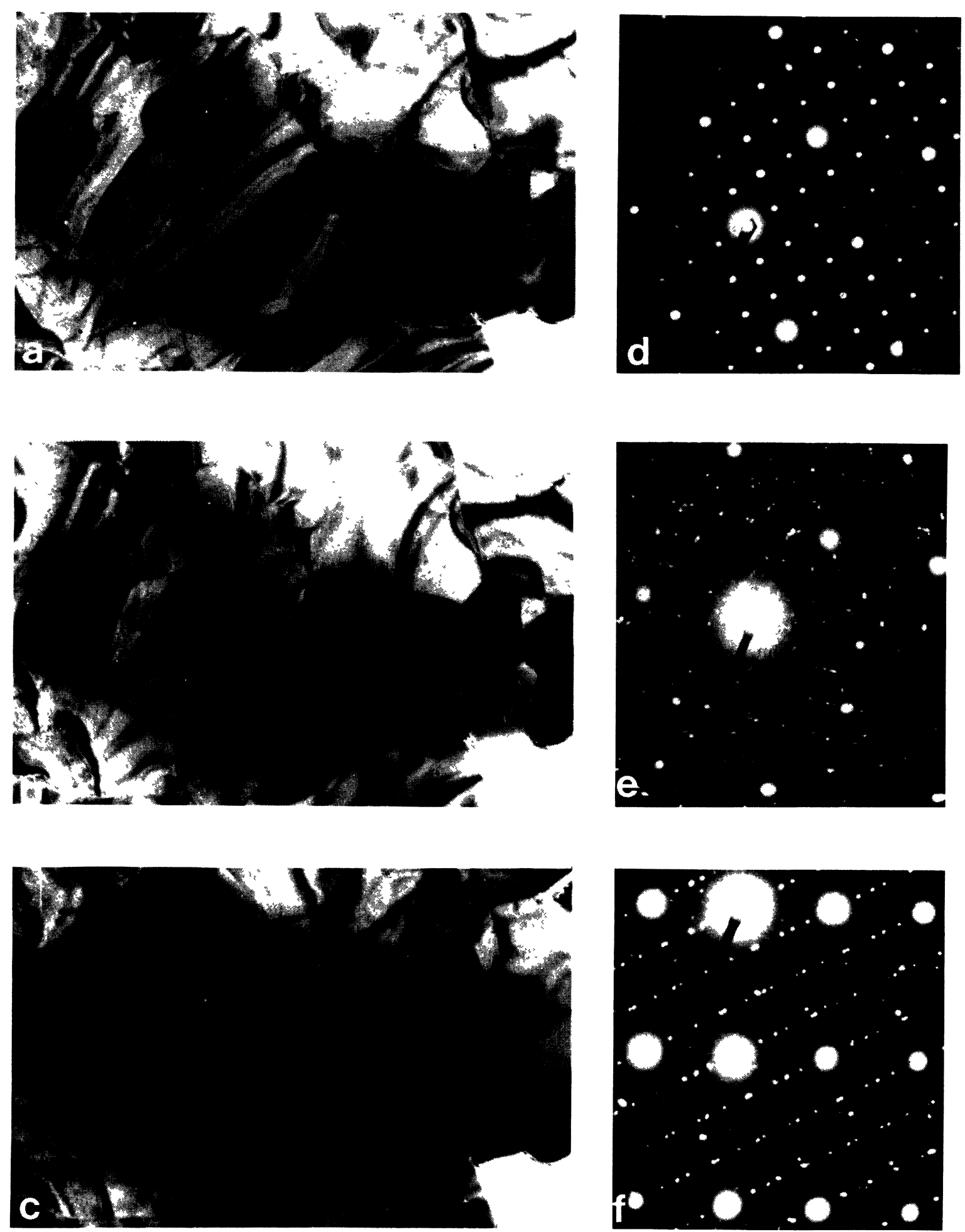

Figs. 1a à 1c. - Modification de l'aspect de cristaux d'oxyde de praséodyme sous l'impact du faisceau d'électrons. [Appearance surface change of thin praseodymium crystals in the course of the electron-beam irradiation.]

Figs. 1d à 1f. - Evolution du diagramme de diffraction électronique d'un de ces cristaux au cours du recuit. [Electron diffraction patterns evolution during annealing (they are relative to the same crystal).] 
de température engendrent des transitions totalement aléatoires et aucun d'eux n'est reproductible. Toutes ces structures sont construites sur la base de la maille fluorine des dioxydes. Elles sont tributaires du réarrangement des ions oxygènes et des lacunes. Mais le mécanisme reste encore inconnu malgré les études de microscopie électronique à haute résolution [6-8]. Certains diagrammes relatifs à la structure $\mathrm{RO}_{2}$ sont incohérents avec le réseau c.f.c. : rapports des distances interréticulaires inexacts et angles différents de $90^{\circ}$. Ceci implique une distortion lors des changements de phase.

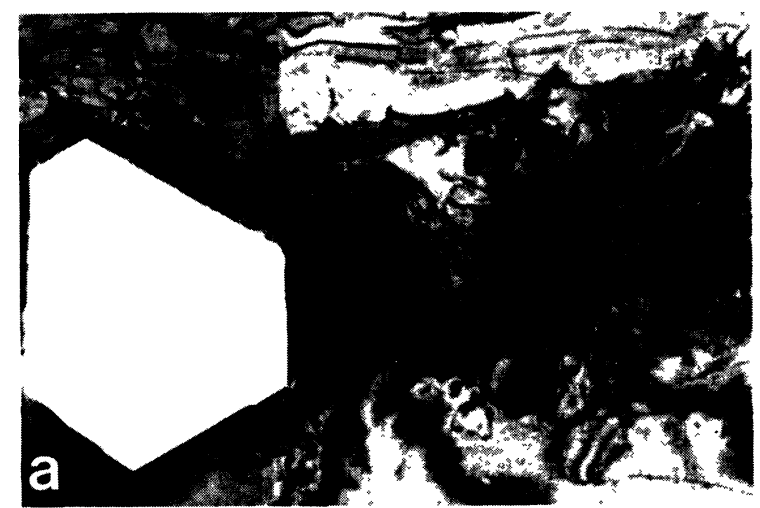

Figs. $2 \mathrm{a}$ et $2 \mathrm{~b}$. - Cristaux de type $\mathrm{B}-\mathrm{Pr}_{2} \mathrm{O}_{3}$ (2b : fond noir). [B- $\mathrm{Pr}_{2} \mathrm{O}_{3}$ (monoclinic) crystals (2b : dark field).]

$$
\begin{array}{cc}
\mathrm{A}-\mathrm{Nd}_{2} \mathrm{O}_{3} & \mathrm{~B}-\mathrm{Nd}_{2} \mathrm{O}_{3} \\
\text { Axe de zone }[001] & \text { Axe de zone [11i] }
\end{array}
$$
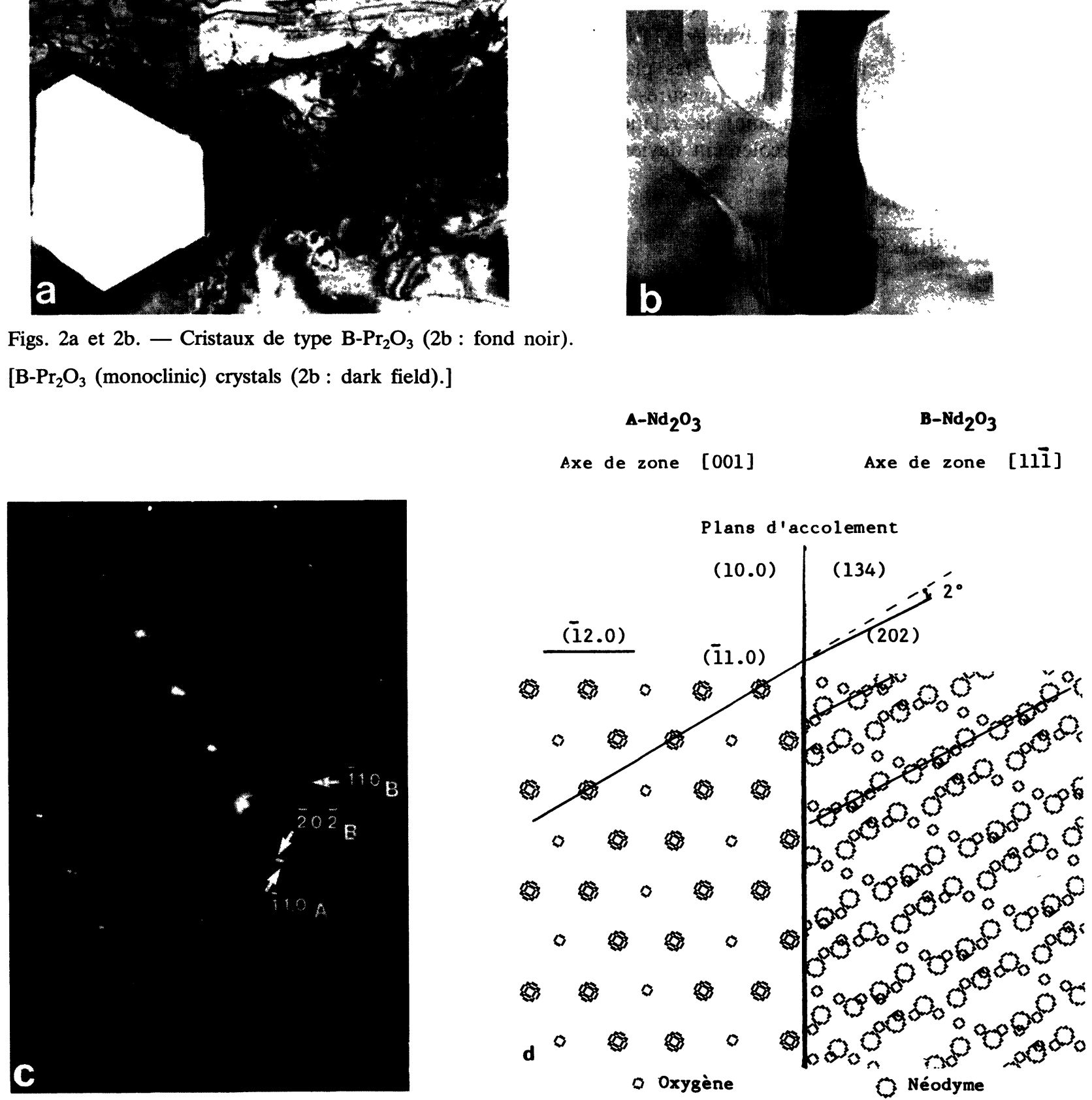

Figs. $2 \mathrm{c}$ et $2 \mathrm{~d}$. - Diagramme de diffraction et projection des structures suivant les axes de zone relatifs à l'épitaxie de deux cristaux de type A- et $\mathrm{B}-\mathrm{Nd}_{2} \mathrm{O}_{3}$.

[Electron diffraction pattern showing the epitaxial mode between two A- and $\mathrm{B}-\mathrm{Nd}_{2} \mathrm{O}_{3}$ crystals.] 
La phase $\mathrm{B}-\mathrm{R}_{2} \mathrm{O}_{3}$ (monoclinique) est très difficile à obtenir, car son domaine de stabilité est très étroit. En couches minces, elle a été observée en épitaxie sur des cristaux de type $\mathrm{A}-\mathrm{R}_{2} \mathrm{O}_{3}$, à haute température, et avec un temps de vie très bref [3]. Dans le cas présent des cristaux de type $\mathrm{B}-\mathrm{R}_{2} \mathrm{O}_{3}$ restent remarquablement stables après formation à haute température. Aléatoirement répartis au sein de la matrice $\mathrm{A}-\mathrm{R}_{2} \mathrm{O}_{3}$, ils présentent une épitaxie aux joints de grains de ces cristaux (Figs. 2a à 2d). Le diagramme de diffraction électronique (Fig. 2c) permet de définir la relation d'épitaxie entre deux cristaux A- et B-. La projection des réseaux obtenue à l'aide d'un microordinateur précise les caractéristiques cristallographiques de l'épitaxie (Fig. 2d). Le rapport entre les distances réticulaires $(\overline{1} 1.0)_{\mathrm{A}}$ et $(202)_{B}$ est 1,046 . Cependant comme les plans ne sont parallèles qu'à environ $2^{\circ}$ près (mesurés sur les taches dédoublées du diagramme) la relation de raccordement sur le plan d'accolement devient :

$$
\frac{d_{202} / \cos (58)}{d_{\overline{1} 1.0} / \cos (60)}=1,013 \text {. }
$$

Un raccordement parfait est obtenu pour un écart angulaire de $1,54^{\circ}$, raisonnable étant donné l'imprécision de la mesure.
4.2 Terbium. - Pour cet élément les sesquioxydes de type C- et $\mathrm{B}-\mathrm{Tb}_{2} \mathrm{O}_{3}$ sont facilement stabilisés en couches minces (3). Dans le cas présent, nous avons observé une épitaxie aux joints de grains des structures $\mathrm{B}-\mathrm{Tb}_{2} \mathrm{O}_{3}$ et $\mathrm{TbO}_{2}[9]$.

\section{Conclusion.}

Les phénomènes de transition de phase et certains modes d'épitaxie dans les oxydes de terres rares sont encore mal compris et donc très difficiles à interpréter dans le cas de couches minces irradiées par un faisceau électronique. On suppose que des ondes de choc générées au sein du matériau entraînent des réarrangements structuraux.

Il n'est malheureusement pas possible de déterminer expérimentalement les effets induits par l'irradiation d'un matériau à l'aide d'un faisceau d'électrons. Nous avons montré [3] que si celui-ci est fortement focalisé les couches minces de sesquioxides de terres rares peuvent, soit former les phases de très hautes températures (proches de $2000 \mathrm{~K}$ ), soit être volatilisées sous forme de molécules RO. La création d'ondes acoustiques provient alors soit du processus d'ablation soit des gradients thermiques à l'intérieur de l'échantillon. La formation de la phase de haute pression $\mathrm{B}-\mathrm{R}_{2} \mathrm{O}_{3}\left(\mathrm{~B}-\mathrm{Pr}_{2} \mathrm{O}_{3}\right.$ et $\mathrm{B}-\mathrm{Nd}_{2} \mathrm{O}_{3}$ n'existent pas dans les conditions P-T normales) montrerait ainsi que le matériau est soumis à de fortes pressions.

\section{Bibliographie}

[1] Gasgnier, M., Schiffmacher, G. et Caro, P., Inorg. Chem. Acta 140 (1987) 79.

[2] Gasgnier, M., Schiffmacher, G., et Caro, P., J. Microsc. Spectrosc. Electron. 13 (1988) 13.

[3] Gasgnier, M., Phys. Status Solidi a 57 (1980) 11.

[4] Gasgnier, M., Schiffmacher, G., Caro, P. et Eyring, L., J. Less. Common Met. 116 (1986) 31.

[5] Gasgnier, M., SChiffmacher, G., Eyring, L. et CARo, P., J. Less-Common Met. 127 (1987) 167.
[6] Tuenge, R. T. et Eyring, L., J. Solid State Chem. 29 (1979) 165.

[7] Tuenge, R. T. et Eyring, L., J. Solid State Chem. 41 (1983) 75.

[8] Eick, H. A., Eyring, L., Summerville, E. and Tuenge, R. T., J. Solid State Chem. 42 (1982) 47.

[9] GAsgnier, M., Schiffmacher, G. and CARo, P., à soumettre à J. Mater. Sci. 